\title{
Effect of cervical sympathetic block on cerebral vasospasm after subarachnoid hemorrhage in rabbits ${ }^{1}$
}

\author{
He Chun-jing', Ou Shan" ${ }^{I I}$ Liu Guo-dongII, Nie Hao-xiong ${ }^{I V}$, Luo Yi-ran ${ }^{I V}$, Feng Ya-ping ${ }^{\mathrm{V}}$ \\ IAssociate Professor, Department of Anesthesiology, People's Hospital of Guizhou Province, China. Acquisition and interpretation of data, statistical \\ analysis. \\ IIPhD, Associate Professor, Department of Anesthesiology, General Hospital of Chengdu Military Command, China. Acquisition and interpretation of \\ data, statistical analysis.

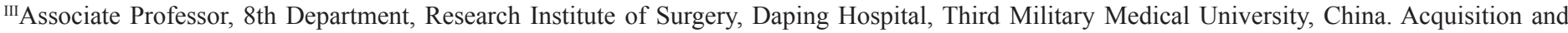 \\ interpretation of data, statistical analysis.

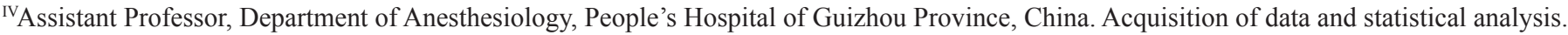

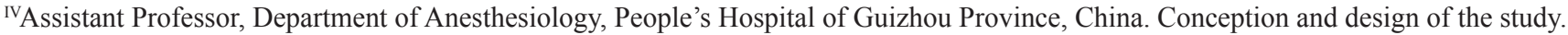 \\ vAssociate Professor, Department of Anesthesiology, People's Hospital of Guizhou Province, China. Conception and design of the study.
}

\section{ABSTRACT}

PURPOSE: Cerebral vasospasm (CVS) is a major complication after subarachnoid hemorrhage (SAH) induced by the rupture of intracranial aneurysms. The aim of the present study was to investigate the effect and mechanism of cervical sympathetic block on cerebral vasospasm of the rabbits after SAH.

METHODS: After successful modeling of cervical sympathetic block, 18 healthy male white rabbits were randomly divided into three groups ( $\mathrm{n}=6$ ), ie, sham operation group (Group A), SAH group (Group B) and SAH with cervical sympathetic block group (Group C). Models of delayed CVS were established by puncturing cisterna magna twice with an injection of autologous arterial blood in Groups B and C. A sham injection of blood through cisterna magna was made in Group A. $0.5 \mathrm{ml}$ saline was injected each time through a catheter for cervical sympathetic block after the first injection of blood three times a day for $3 \mathrm{~d}$ in Group B (bilateral alternating). $0.5 \mathrm{ml}$ of $0.25 \%$ bupivacaine was injected each time through a catheter for cervical sympathetic block after the first injection of blood three times a day for $7 \mathrm{~d}$ in Group B. $2 \mathrm{ml}$ venous blood and cerebrospinal fluid were obtained before (T1), $30 \mathrm{~min}$ (T2) and $7 \mathrm{~d}$ (T3) after the first injection of blood, respectively, and conserved in a low temperature refrigerator. Basilar artery value at T1, T2 and T3 was measured via cerebral angiography. The degree of damage to nervous system at $\mathrm{T} 1$ and $\mathrm{T} 3$ was recorded.

RESULTS: There was no significant difference in diameter of basilar artery at $\mathrm{T} 1$ among three groups. The diameters of basilar artery at T2 and T3 of Groups B and C were all smaller than that in Group A, which was smaller than Group C, with a significant difference. There was no significant difference in NO and NOS in plasma and cerebrospinal fluid among three groups. The NO and NOS contents at T2 and T3 of Groups B and C were all lower than Group A; Group C was higher than Group B, with a significant difference. The nerve function at T3 of Groups B and C were all lower than Group A and that of Group C higher than Group B, with a significant difference. CONCLUSION: Cervical sympathetic block can relieve cerebral vasospasm after subarachnoid hemorrhage and increase NO content and NOS activity in plasma and cerebrospinal fluid to promote neural functional recovery.

Key words: Sympathectomy. Subarachnoid Hemorrhage. Vasospasm, Intracranial. Rabbits. 


\section{Introduction}

Cerebral vasospasm (CVS) is a major complication after subarachnoid hemorrhage (SAH) induced by rupture of intracranial aneurysms. ${ }^{1}$. Over $50 \%$ patients with $\mathrm{SAH}$ have symptomatic CVS (accounting for $40 \%$ ) that is the main cause of disability and mortality. Over $20 \%$ patients without treatment were left with permanent neurologic impairment or even died ${ }^{2-}$ 5. Despite extensive clinical and experimental studies, the pathogenesis of cerebra vasospasm is still controversial and poorly understood. The cause of vasospasm is presently considered to be multifactorial. In the presence of CVS, current management consists of hypertension, high blood volume and blood high dilution $(3 \mathrm{H})$ therapy and neuroradiological procedures ${ }^{6-13}$. The results of these treatment options have been hampered by some technical limitations and medical complications. Therefore, understanding of the pathological and physical mechanism and exploration of effective therapy have become the hot topic in current research. Cervical sympathetic block is a safe and effective therapy ${ }^{14-15}$ with little side effect. Cervical sympathetic block can significantly dilate cerebral vessels, increase cerebral blood velocity, regulate the imbalance with endothim (ET) and calcitonin gene related protein (CGRP) and excessive expression of neurons' heat shock protein 70 after global cerebral ischemia-reperfusion injury in rabbits, improve the immune function of erythrocyte of patients with cerebral infarction, regulate the content of substance causing systolic and diastolic vessels in serum such like NO and NOS, and promote neural functional recovery. At present, there still exists argument on alleviative role of cervical sympathetic block on CVS after SAH and the role of cervical sympathetic block in regulating substances causing systolic and diastolic vessels to protect the brain. Wang et al. ${ }^{16}$ found that the upper thoracic epidural external sympathetic block with $0.5 \%$ lidocaine dilated the cerebral vessels of rabbits after SAH, increased cerebral blood flow and relieved the nervous dysfunction, which was effective to CVS after SAH. However, Treggiari et al. ${ }^{17}$ studied effect of the transaction of sympathetic nerves after SAH on vascular smooth muscles and the mechanism by recording the response of carotid artery in vitro to norepinephrine, 5-hydroxytryptamine and endothelin. The results showed that one week after $\mathrm{SAH}$, the supersensitivity of carotid artery and cerebral artery caused by SAH induced CVS. Neither the contractile response of cervical arteries to epinephrine and 5-hydroxytryptamine nor CVS mediated by ET-1 could be relieved or prevented by the transaction of sympathetic nerves. The inconsistent result may be related with the opportunity of transaction and blockage of cervical sympathetic nerves. The aim of the present study was to investigate the effect and mechanism of cervical sympathetic block on CVS of rabbits after SAH.

\section{Methods}

All procedures were approved by Animal Research Committee of People's Hospital of Guizhou Province, China. Healthy SD New Zealand rabbits (male, weighing $2-2.5 \mathrm{~kg}$, aged 6-7 months) were provided by the Animal Laboratory of Third Military Medical University, Chongqing.

(1) Establishment of experimental models. The model of cervical sympathetic block: $3 \%$ pentobarbital sodium $(1 \mathrm{ml} / \mathrm{kg})$ was injected though ear vein of healthy rabbits. After anesthesia, an incision was made at the neck, placing catheters on both sides respectively, for a blunt separation along cervical artery to cervical sympathetic. One end of the catheter was placed and fixed near the cervical sympathetic; the other end along the spine through the skin of nape was fixed to expose a $2 \mathrm{~cm}$ long section for a local anesthesia. The rabbits with no infected incision were prepared for experiments after being fed for one week.

The double-hemorrhage model of SAH was used in this experiment ${ }^{18}$. After successful modeling of cervical sympathetic block, 18 healthy male rabbits were randomly divided into three groups $(n=6)$, ie, sham operation group (Group A), SAH group (Group B) and SAH with cervical sympathetic block group (Group C). Models of delayed CVS were established by puncturing cisterna magna twice with an injection of autologous arterial blood $(0.8 \mathrm{ml} /$ $\mathrm{kg}$, at interval of 48 hours) in Groups B and C. A sham injection of blood through cisterna magna (equivalent normal saline at the same time) was made in Group A. Normal saline for $0.5 \mathrm{ml}$ was injected each time through a catheter for cervical sympathetic ganglia block after the first injection of blood three times a day for three days in Group B (bilateral alternating). Bupivacaine at $0.25 \%$ for $0.5 \mathrm{ml}$ was injected each time through a catheter for cervical sympathetic ganglia block after the first injection of blood three times a day for seven days in Group C (bilateral alternating).

Pentobarbital sodium $(1 \mathrm{ml} / \mathrm{kg})$ at $3 \%$ was injected through ear vein at T1, T2 and T3 to induce anesthesia. Then, the rabbits were placed in a supine position, and a 5-F catheter (Terumo Corp., Japan) was inserted selectively into the aortic arch through a femoral artery by the Seldinger method. Angiograms of the basilar artery were obtained by injection of $5 \mathrm{ml}$ Omnipaque contrast medium for two seconds at a pressure of 50 psi. The speed of digital image acquisition was six frames per second. The angiograms were transferred to an analytic processing system, and the diameter of the basilar artery was measured at five points (at 
the midpoint of the basilar artery, at $1 \mathrm{~mm}$ central and peripheral from the midpoint, and at $2 \mathrm{~mm}$ central and peripheral from the midpoint). The mean diameter at these five points was then determined. All angiograms were obtained by one investigator and the diameters of the basilar artery were measured by a colleague working in a blinded fashion. Angiograms were performed at T1, $\mathrm{T} 2$ and $\mathrm{T} 3$.

Venous blood and cerebrospinal fluid for $2 \mathrm{ml}$ at $\mathrm{T} 1$, $\mathrm{T} 2$ and T3 were obtained respectively and conserved in a lower temperature refrigerator $\left(-80^{\circ} \mathrm{C}\right)$. The degree of nervous system damage at $\mathrm{T} 3$ was recorded.

(2) Neurological score. The discrimination of changes in neurological state was conducted with Otsuji' method 19 and neurological score at T3 was graded (Chart 1).

CHART 1 - Classification standard for neurological state of experimental animals.

\begin{tabular}{|ll|}
\hline Grade & Symptoms of nerve damage \\
\hline Grade I & None \\
Grade II & $\begin{array}{l}\text { Mild or not obvious (suspicious) symptoms of nerve } \\
\text { damage }\end{array}$ \\
Grade III & Moderate symptoms of nerve damage \\
Grade IV & Severe symptoms of nerve damage \\
\hline
\end{tabular}

\section{Statistical analysis}

Statistical software SAS was used for analysis. All measurement data were presented as mean \pm standard deviation. Group $t$-test was used for comparison among groups, variance analysis of repeated measurement data for intra-group comparison and Chi-square test for enumeration data. $\mathrm{p}<0.05$ indicates a significant difference.

\section{Results}

There were no significant differences in each group in terms of basilar artery diameter, NO content and NOS activity in the serum and cerebrospinal fluid at T1 $(\mathrm{p}>0.05)$. The rabbit doublehemorrhage models were established successfully. In Group B and
Group C, the basilar artery vasospasm was readily apparent at T2 and T3 (Figure 1 from a to f). There was no significant difference in diameter of basilar artery at T1 among three groups. Basilar artery vasospasm at different degrees was seen at T2 and T3 in all groups (Figure 1 from a to $\mathbf{b}$ ).
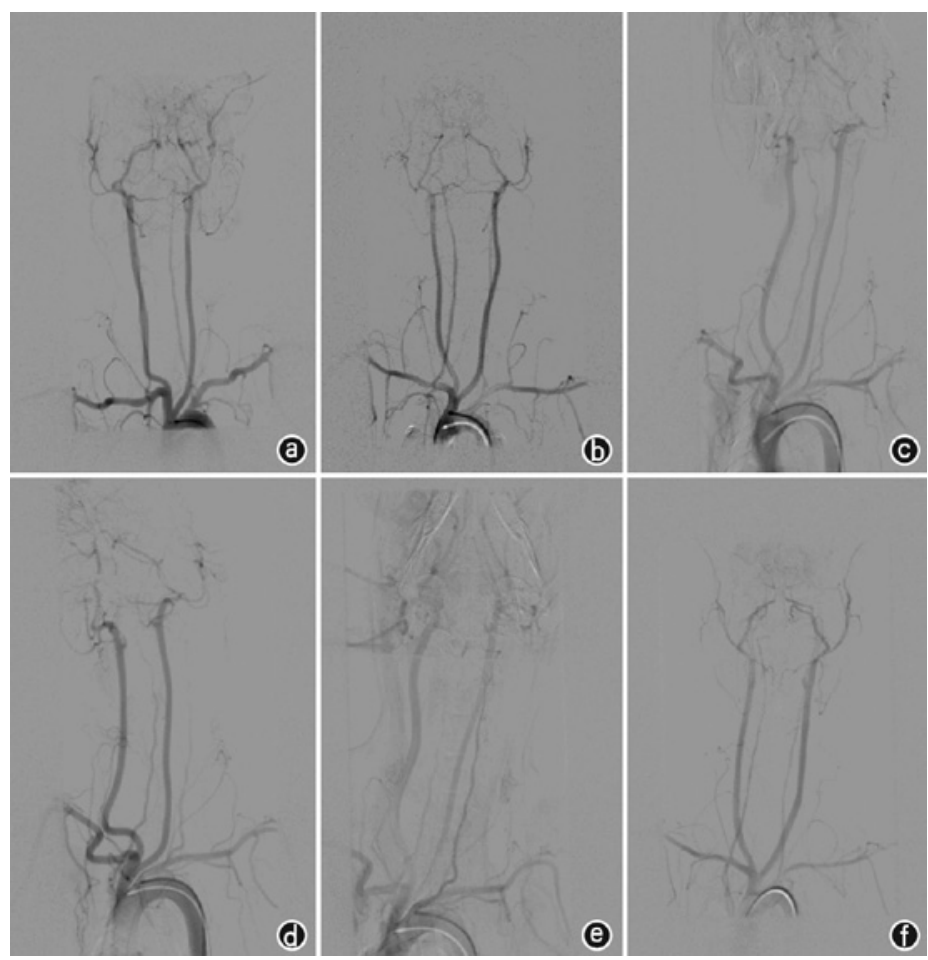

FIGURE 1 - The changes of the basilar artery vasospasm. Before SAH, there showed no basilar artery vasospasm in Group A (a), Group B (b) and Group C (c). At day 7 after SAH, various degrees of the basilar artery vasospasm was found in Group A (d), Group B (e) and Group C (f), with more obvious vasospasm in Group A than that in Groups B and C.

The diameter of basilar artery at T2 and T3 in Groups B and $\mathrm{C}$ were all smaller than that in Group A. While the diameter of basilar artery at T2 and T3 in Group B was smaller than that in Group C, with a significant difference $(p<0.01<)$.Cervical sympathetic block significantly decreased the narrowing of the basilar artery after SAH (Table 1).

There was no significant difference in NO content and NOS activity in serum and cerebrospinal fluid at T1 among three groups. NO content and NOS activity at T2 and T3 were lower than that at T1 in all groups; while NO content and NOS activity in Group $\mathrm{C}$ was lower than that in Group A but higher than that in Group B, with a significant difference $(\mathrm{p}<0.01)$ (Table 1). 
TABLE 1 - Changes of basilar artery diameter, NO content and NOS activity in serum and cerebrospinal fluid at different time points.

\begin{tabular}{|c|c|c|c|c|}
\hline Index & Group & $\mathrm{T} 1$ & $\mathrm{~T} 2$ & $\mathrm{~T} 3$ \\
\hline \multirow{3}{*}{$\begin{array}{l}\text { Diameter of } \\
\text { basilar artery } \\
(\mathrm{mm})\end{array}$} & A & $0.7 \pm 0.02$ & $0.58 \pm 0.04^{*}$ & $0.67 \pm 0.03$ \\
\hline & B & $0.7 \pm 0.02$ & $0.15 \pm 0.05^{* \#}$ & $0.34 \pm 0.06^{* \#}$ \\
\hline & $\mathrm{C}$ & $0.7 \pm 0.03$ & $0.42 \pm 0.06^{* \# \Delta}$ & $0.57 \pm 0.06^{*} \# \Delta$ \\
\hline \multirow{3}{*}{$\begin{array}{l}\text { NO in serum } \\
(\mu \mathrm{mmol} / \mathrm{L})\end{array}$} & A & $54.97 \pm 4.17$ & $40.07 \pm 6.97^{*}$ & $57.08 \pm 4.65$ \\
\hline & B & $54.45 \pm 4.33$ & $21.08 \pm 5.98^{* \#}$ & $28.38 \pm 5.82^{*}$ \# \\
\hline & $\mathrm{C}$ & $54.73 \pm 8.74$ & $50.58 \pm 6.26^{*}$ \# & $55.52 \pm 8.25^{* \# \Delta}$ \\
\hline \multirow{3}{*}{$\begin{array}{c}\text { NO in } \\
\text { cerebrospinal } \\
\text { fluid } \\
(\mu \mathrm{mmol} / \mathrm{L})\end{array}$} & A & $69.77 \pm 2.52$ & $67.22 \pm 3.22$ & $69.25 \pm 2.62$ \\
\hline & B & $69.82 \pm 3.11$ & $25.4 \pm 4.58^{* \#}$ & $25.42 \pm 4.47^{* \#}$ \\
\hline & $\mathrm{C}$ & $69.8 \pm 2.58$ & $36.65 \pm 6.07^{*}$ \# & $40.33 \pm 4.86^{* \# \Delta}$ \\
\hline \multirow{3}{*}{$\begin{array}{l}\text { NOS in } \\
\text { serum } \\
(\mathrm{U} / \mathrm{ml})\end{array}$} & A & $54.87 \pm 4.09$ & $55.17 \pm 33.31$ & $53.25 \pm 3.77$ \\
\hline & B & $55.03 \pm 4.61$ & $21.02 \pm 3.13^{* \#}$ & $22.38 \pm 1.90^{*}$ \# \\
\hline & $\mathrm{C}$ & $54.98 \pm 3.03$ & $36.83 \pm 3.33^{*} \# \Delta$ & $39.8 \pm 4.01^{* \# \Delta}$ \\
\hline \multirow{3}{*}{$\begin{array}{c}\text { NOS in } \\
\text { cerebrospinal } \\
\text { fluid } \\
(\mathrm{U} / \mathrm{ml}) \\
\end{array}$} & A & $57.45 \pm 4.83$ & $51.42 \pm 5.47^{*}$ & $50.12 \pm 5.67^{*}$ \\
\hline & B & $57.65 \pm 4.36$ & $21.37 \pm 3.43^{* \#}$ & $22.5 \pm 3.18^{* \#}$ \\
\hline & $\mathrm{C}$ & $56.9 \pm 4.47$ & $35.42 \pm 4.63^{*}$ \# & $38.83 \pm 4.15^{*}$ \# \\
\hline
\end{tabular}

Notes: Compared with $\mathrm{T}_{1}$ : " $\mathrm{p}<0.01$; Compared with group $\mathrm{A}$ : ${ }^{*} \mathrm{p}<0.01$; Compared with group B: ${ }^{\triangle} \mathrm{p}<0.01$.

The neurological function at T3 in Groups B and $\mathrm{C}$ were all lower than that in Group A; while the neurological function at T3 in Group C was higher than that in Group B, with significant difference (Table 2).

TABLE 2 - Neurological function of rabbits in all groups post treatment.

\begin{tabular}{ccccc}
\hline Groups & I & II & III & IV \\
\hline A & 6 & 0 & 0 & 0 \\
B & $0^{*}$ & 0 & $2^{*}$ & $4^{*}$ \\
C & $0^{* \#}$ & $2^{* \#}$ & $3^{* \#}$ & $1^{* \#}$ \\
\hline
\end{tabular}

Notes: Compared with Group A: " $\mathrm{p}<0.01$; Compared with Group B: ${ }^{*}<<0.01$.

The animals in our experiment showed no obvious abnormal symptoms associated with the adverse effects of cervical sympathetic block. This results demonstrated that cervical sympathetic block had therapeutic efficacy in the experimental CVS.

\section{Discussion}

The present study shows that a noninvasive procedure of temporary chemical sympathectomy contributes to prevention and treatment of CVS. The rationale for this procedure is that the blockage of sympathetic nerve activity or reversal of overactivity may dilate intracerebral vessels. Nitric oxide (NO) plays an important role in the regulation of hemostasis. It is believed that vasospasm is resulted from the decreased availability of NO and the reduced endothelial and neuronal NOS-mediated relaxation of large conductive cerebral arteries ${ }^{20-22}$. In this study, the NO content and NOS activity in the serum and cerebrospinal fluid of Groups $\mathrm{B}$ and $\mathrm{C}$ after SAH were significantly lower than that before $\mathrm{SAH}$. The explanation for decreased level of NO in the present study is that the presence of blood around the major cerebral arteries caused morphological changes in the vessels, including the endothelial cells $^{23}$. This endothelial injury impairs the equilibrium between $\mathrm{NO}$ and prostacylin whose balance is critical for the maintenance of vascular tone ${ }^{24,25}$. Previous reports have indicated that CVS is observed as early as 30 minutes (the acute phase) ${ }^{26}$ after SAH, and reaches a peak at days 3-7 (the chronic phase) ${ }^{27}$. Among several animal models of CVS, the double-hemorrhage model was considered to resemble more closely the human pathological features of $\mathrm{CVS}^{27}$. Therefore, we adopted to successfully establish this model of CVS. In the double-hemorrhage model, it has been reported that there are a variety of morphological changes in the arteries in spasm, such as endothelial cell vacuolization, disruption of the internal elastic lamina, and so on. In the present study, it is reasonable to consider that the inhibitory effect of cervical sympathetic block on the experimental CVS after SAH may have contributed to the increase in NO content and NOS activity in the serum and cerebrospinal fluid. It may be due to that postganglionic fiber of cervical sympathetic ganglia regulated and excreted NOS. It has proved that use of sympathetic ganglion inhibitor quaternary ammonium at stellate ganglion can increase the excretion of NOS at nerve endings ${ }^{28-30}$.

\section{Conclusion}

Cervical sympathetic block can relieve cerebral vasospasm and increase NO content and NOS activity in plasma and cerebrospinal fluid to promote neural function recovery_after subarachnoid hemorrhage in rabbits.

\section{References}

1. Kassekk NF, Sasaki T, Colohan AR, Nazar G. Cerebral vasospasm following aneurysmal subarachnoid hemorrhage. Stroke. 1985;16:562-72.

2. Donch NW. Therapeutic approaches to vasospasm in subarachnoid hemorrhage. Curr Opin Crit Care. 2002;8:128-33.

3. Wang XY, Wang QX, Yang G, Fu NA, Liu JY, Luo ME, Liu AY. Effect of stellate ganglion block on the content of substance $\mathrm{P}$ in 
spine of rabbit with formalin-induced pain. Chin J Anesthesiol. 2002;5:301-3.

4. Buchanan KM, Elias LJ, Goplen GB. Differing perspectives on outcome after subarachnoid hemorrhage: the patient, the relative, the neurosurgeon. Neurosurgery. 2000;46:831-8.

5. Carter BS, Buckley D, Ferraro R, Rordorf G, Ogilvy CS. Factors associated with reintegration to normal living after subarachnoid hemorrhage. Neurosurgery. 2000;46:1326-33.

6. Levy ML, Day JD, Zelman V, Giannotta SL. Cardiac performance enhancement and hypervolemic therapy. Neursurg Clin N Am. 1994;5:725-39.

7. Newell DW, Eskridge JM, Mayberg MR, Grady MS, Winn HR. Angioplasty for the treatment of symptomatic vasospasm following subarachnoid hemorrhage. J Neurosurg. 1989;71:654-60.

8. Findlay JM. Current management of aneurysmal subarachnoid hemorrhage guidelines from the Canadian Neurosurgical Society. Can J Neurol Sci 1997;24:161-70.

9. Firlik AD, Kaufmann AM, Jungreis CA, Yonas H. Effect of transluminal angioplasty on cerebral blood flow in the management of symptomatic vasospasm following aneurysmal subarachnoid hemorrhage. J Neurosurg. 1997;86:830-9.

10. Milburn JM, Moran CJ, Cross DT3, Diringer MN, Pilgram TK, Dacey RG Jr. Increase in diameters of vasospastic intracranial arteries by intraarterial papaverine administration. J Neurosurg. 1998;88:38-42.

11. Bejjani GK, Bank WO, Olan WJ, Sekhar LN. The efficacy and sagety of angioplasty for cerebral vasospasm after subarachnoid hemorrhage. Neurosurgery. 1998;42:979-86.

12. Eskridge JM, McAuliffe W, Song JK, Deliganis AV, Newell DW, Lewis DH, Mayberg MR, Winn HR. Balloon angioplasty for the treatment of vasospasm: results of first 50 cases. Neurosurgery. 1998;42:510-6.

13. Muizelaar JP, Zwienenberg M, Rudisill NA, Hecht ST. The prophylactic use of transluminal balloon angioplasty in patients with Fisher grade 3 subarachnoid hemorrhage:a pilot study. J Meirpsirg. 1999;91:51-8.

14. Quan SB, Liu JY, Wang QX, Yang GA, Fu NA. Effect of stellate ganglion block on the genes of endothelin and calcitonin related peptides in rabbits with global cerebral ischemia-reperfusion injury. J Clin Anesthesiol. 2005;4:260-2.

15. HE CJ, Yu Q, Feng YP, Liang DY, Ran Y. Effect of stellate ganglion block on immune function of akaryocyte of patients with acute cerebral infarction. Chin J Anesthesiol. 2007;7:640-2.

16. Wang HM, Li ZX, Li EY, Dong WY, Zhang YA, Qun RH, Li GL. Effect of high thoracic epidural symathetic block on cerebral vasospasm after subarachnoid hemorrhage in rabbits. Chin J Anesthesiol. 2008;28:234-7.

17. Treggiari MM, Romand JA, Martin JB, Reverdin A, Rufenacht DA, Detribolet N. Cervical sympathetic block to reverse delayed ischemic neurological deficits after aneurismal subarachnoid hemorrhage. Stroke. 2003;34:961-7.

18. Wang L, Shi JX, Yin HX, Ma CY, Zhang QR. The influence of subarachnoid hemorrhage on neurons: an animal model. Ann Clin Lab Sci. 2005;35(1):79-85.

19. Otsuji T, Endo S, Hirashima Y, Nishijima M, Takaku A. An experimental model of symptomatic vasospasm induced by oxyhemoglobin in rabbits. Stroke. 1994;25(3):657-62.

20. Jung CS, Oldfield EH, Harvey-White J, Espey MG, Zimmermann M, Seifert V, Pluta RM. Association of an endogenous inhibitor of nitric oxide synthase with cerebral vasospasm in patients with aneurysmal subarachnoid hemorrhage. J Neurosurg. 2007;107:94550 .

21. Iuliano BA, Pluta RM, Jung C, Oldfield EH. Endothelial dysfunction in a primate model of cerebral vasospasm. J Neurosurg. 2004;100:287-94.

22. Pluta RM, Thompson BG, Dawson TM, Snyder SH, Boock RJ, Oldfield EH. Loss of nitric oxide synthase immunoreactivity in cerebral vasospasm. J Neurosurg. 1996;84:648-54.

23. Iadecola C. Bright and dark sides of nitric oxide in ischemic brain injury. Trends Neurosci. 1997;20:132-49.

24. Clower BR, Yamamoto Y, Cain L, Haines DE, Smith RR. Endothelial injury following experimental subarachnoid hemorrhage in rats: effects on brain blood flow. Anat Rec. 1994;240:104-14.

25. Iuliano BA, Pluta RM, Jung C, Oldfield EH. Endothelial dysfunction in a primate model of cerebral vasospasm. J Neurosurg. 2004;100:287-94.

26. Gioia AE, White RP, Bakhtian B, Robertson JT. Evaluation of the efficacy of intrathecal nimodipine in canine models of chronic cerebral vasospasm. J Neurosurg. 1985;62:721-8.

27. Megyesi JF, Vollrath B, Cook DA, Findlay JM. In vivo animal models of cerebral vasospasm: a review. Neurosurgery. 2000;46:448-60.

28. He SH, Wang QX, Wang YL, Liu JY. Effect of stellate ganglion block on endothelial nitric oxide synthase and pulmonary arterial pressure of rabbits with anoxic pulmonary hypertension. Chin J Anesthesiol. 2004;24:190-3.

29. Yamada K, Kushik K, Yamada H, Katsuragi T, Farukawa T, Noguchi $\mathrm{H}$, Ono N. Contribution of nitric oxide to the presynaptic inhibition by endothelin ETB receptor of the canine stellate ganglionic transmission. J Pharmacol Exp Ther.1999;290:1175-81.

30. He CJ, Li XS, Ran Y, Feng YP. Effects of stellate ganglion block on serum NO level and NOS activity in diabetic patients with Bell's palsy. Chin J Anesthesiol. 2007;27:817-9.

\section{Correspondence:}

\section{Ou Shan}

Department of Anesthesiology, General Hospital of Chengdu Military Command

Chengdu 600041, China

Phone: +86-13981845049

Hcj777330@sina.com

Received: September 11, 2012

Review: November 14, 2012

Accepted: December 12, 2012

Conflict of interest: none

Financial source: National Natural Science Foundation of China (No. 81200964 and No. 31171069)

'Research performed at Department of Anesthesiology, People's Hospital of Guizhou Province and Department of Anesthesiology, General Hospital of Chengdu Military Command, Chengdu, China. 